\title{
Effect of Supplementing Artificial Diet with Certain Antiseptics on The Growth and Productivity of The Silkworm Bombyx mori L.
}

\author{
Wagiha H. Yehia, Magda M. Abd El-Aziz and El-Karaksy, I. A ${ }^{1}$
}

\begin{abstract}
In an attempt to achieve a better diet for rearing larvae of the silkworm Bombyx mori and increase its productivity, different antiseptics were added to a recommended basic semi - artificial diet to prevent contamination with yeasts and molds. The tested antiseptics were potassium sorbate, propionic acid and delvocid. The results indicated that all these materials were effective in extending the shelf life of the diet as well as improving the rate of growth and productivity of silkworm B. mori. The maximum improvement in all tested criteria was occurred with supplementing delvocid to the diet, in comparison to the other tested materials.
\end{abstract}

\section{INTRODUCTION}

The ingredients of artificial diet are studied mainly according to feeding habits and the demand for the growth and development and cocoon quality and quantity of silkworm Bombyx mori L. (Ito, 1981). Rearing of the silkworm on artificial diet, not aseptically, had been widely carried out in certain countries both on a small and a large scale. So, supply of the diet every two or three days is sufficient to support normal growth, since decomposition of the diet can be prevented to some extent with the aid of antiseptics and/or antibiotics (Cai and Li, 1980). Molds as well as yeasts are the most important cause of artificial diet spoilage.

In his continuous studies on the development of artificial diet and its application in sericulture, Ito (1981) found that sodium dihydroacetic acid was considered as antiseptics in low concentrations. Brummer and Stephan (1980) used sorbic acid and sodium, potassium and calcium sorbate as mold inhibitors in the dough of bread.

Accordingly, the present work was carried out to demonstrate the effect of certain antiseptics on extending the shelf life of the artificial diet as well as their effects on the growth and productivity of the silkworm B. mori.

\section{MATERIALS AND METHODS}

\section{Silkworm races:}

The Chinese F1 hybrid 9F7X of mulberry silkworm Bombyx mori L. was used during the present study. The larvae were reared under the laboratory hygrothermic conditions of $26.5 \pm 1.2$ ?c and $77.0 \pm 2 \%$ R.H. and provided with suitable amount of clean fresh mulberry leaves until the fourth moulting. Only the fifth larval instar was used in the experiment.

\section{Basic ingredients of artificial diet:}

As shown in Table, 1 the semi - artificial diet used was as that which previously described by Fukuda (1963) and Ito and Inokuchi (1992).

\section{Antiseptics used:}

The used substances as antiseptics in this study and their concentrations were chosen on the basis of previous nutritional studies of silkworms as detailed in the available literature in which are represented in four substances as follows:

1- Sodium Dihydroacetic acid (0.54g.): was used as a control treatment (Table, 1).

2- Propionic acid $0.2 \%(0.15 \mathrm{ml})$ : Bertleff et al (2007) reported that propionic acid inhibits the growth of mold and some bacteria. As a result, propionic acid was used as a preservative for both animal feed and food for human consumption.

3- Potassium sorbate $(0.1$ g.): is a natural organic compound used as a preservative in food to prevent the growth of mold, yeast and fungi (Miao and Jiang, 2001)

4- Delvocid (0.1g): is an antimycotic of which natamycin is the active compound. Delvocid is active against all yeasts and molds. In addition it improved quality and appearance of the products treated (Gomaa, 1987).

\section{Experimental design:}

The experiments were designed on the basis of four treatments. Each treatment was represented in one of the aforementioned tested antiseptic materials supplemented to the basic artificial diet (Table 1).

\section{Storage of tested diets:}

Each treatment of prepared tested artificial diet was divided into two sets each of which was stored at room temperature of $24 \pm 1.5 ? \mathrm{c}$ and in the refrigerator at 6?c.

\section{Shelf life of tested artificial diet:}

\footnotetext{
${ }^{1}$ Department of sericulture, Plant Protection Res. Inst.,

Agric. Res. Center.

Received March21, 2009, Accepted March28, 2009
} 
The treatments of artificial diet of both sets mentioned before were daily examined for the appearance of mold growth. The shelf life was indicated as duration (in days) where the diet surface was free of mold growth.

\section{Rearing method:}

The fifth instar larvae were kept on the sliced artificial diet directly and the diet was given once daily. Each treatment was replicated three times and each replicate contained $1505^{\text {th }}$ instar larvae.

\section{The studied biological parameters:}

To estimate the effect of studied antiseptic materials on the growth and productivity of the silkworms, the developmental duration of larval and pupal stages were recorded. Fresh weights of mature larvae, silk glands, pupae, fresh cocoons and cocoon shells were measured. The deposited eggs per female moth were counted and recorded. During the experiments the mortality percentage for each run treatment was calculated.

\section{Statistical analysis:}

The data were statistically analyzed using "F" test and L.S.D. to check the significance between treatments.

\section{RESULTS AND DISCUSSIONS}

Effect of tested antiseptics on the shelf life of the artificial diet:

The results presented in Table, 1 show that the shelf life of the tested artificial diet was markedly affected by the storage temperature as well as the presence of the added antiseptics. Delvocid and potassium sorbate showed longer shelf life being 13 and 9 days respectively at the low temperature. The

Table 1. Basic ingredients of used artificial diet

\begin{tabular}{lc}
\hline Ingredient & Quantity $(\mathbf{g .})$ \\
\hline Mulberry leaf powder & 55 \\
Soybean powder & 10 \\
Agar & 15 \\
Sugar & 10 \\
Ascorbic acid & 1.0 \\
Citric acid & 1.5 \\
Sodium Dihydroacetic acid & 0.54 \\
Water & $270 \mathrm{ml}$. \\
\hline
\end{tabular}

Table 2. Shelf life of artificial diet supplemented with different tested antiseptics

\begin{tabular}{ccc}
\hline & \multicolumn{3}{c}{ Storage temperature } \\
\cline { 2 - 4 } Antiseptics & \multicolumn{2}{c}{ Foom temp. 26.5 \pm 1.2?c } \\
\cline { 2 - 4 } & 13 & Shelf life (days) \\
Delvocid & 9 & 7 \\
Potassium sorbate & 6 & 6 \\
Propionic acid & 4 & 4 \\
Sodium dihydroacetic acid & & 3 \\
\hline
\end{tabular}

corresponding duration at room temperature was 7 and 6 days. As for propionic acid the shelf life was slightly elongated in comparison to the supplementation of sodium dihydroacetic acid (Table, 2).

In agreement with these results, Gomaa (1987) mentioned that the addition of delvocid as well as propionic and sodium diacetate to the dough increased the duration of bread storage. According to Miao and Jiang (2001), adding antiseptics is important to prevent the artificial diet of B.mori from devaluation. They also stated that sorbic acid $10 \%$, propionic acid $15 \%$ or bavistin $0.05 \%$ extended the shelf life of the diet more than ten days.

Effect of tested antiseptics on the weights of larvae, silk glands and pupae:

Statistical analysis of the data (Table 3) shows the highly significant differences between the calculated average weights of the mature larvae, silk glands and pupae which obtained after raising $5^{\text {th }}$ instar larvae on artificial diet supplemented with the different tested antiseptic materials. The maximum improvement in larval, silk gland and pupal weights was recorded in case of supplementing delvocid. The rate of increase was about $16.53,42.14$ and $16.97 \%$ more than the control treatment which was supplemented with sodium dihydroacetic acid (1.658, 0.178 and $1.173 \mathrm{~g}$., respectively). These averages were slightly decreased in the treatment of potassium sorbate, being of 1.825 , 0.214 and $1.357 \mathrm{~g}$, respectively but still significantly more than the control treatment (Table 3 ). The lightest weights of the same characteristics were obtained when larvae were raised on the diet supplemented with propionic acid which was almost statistically equal to the control treatment. 


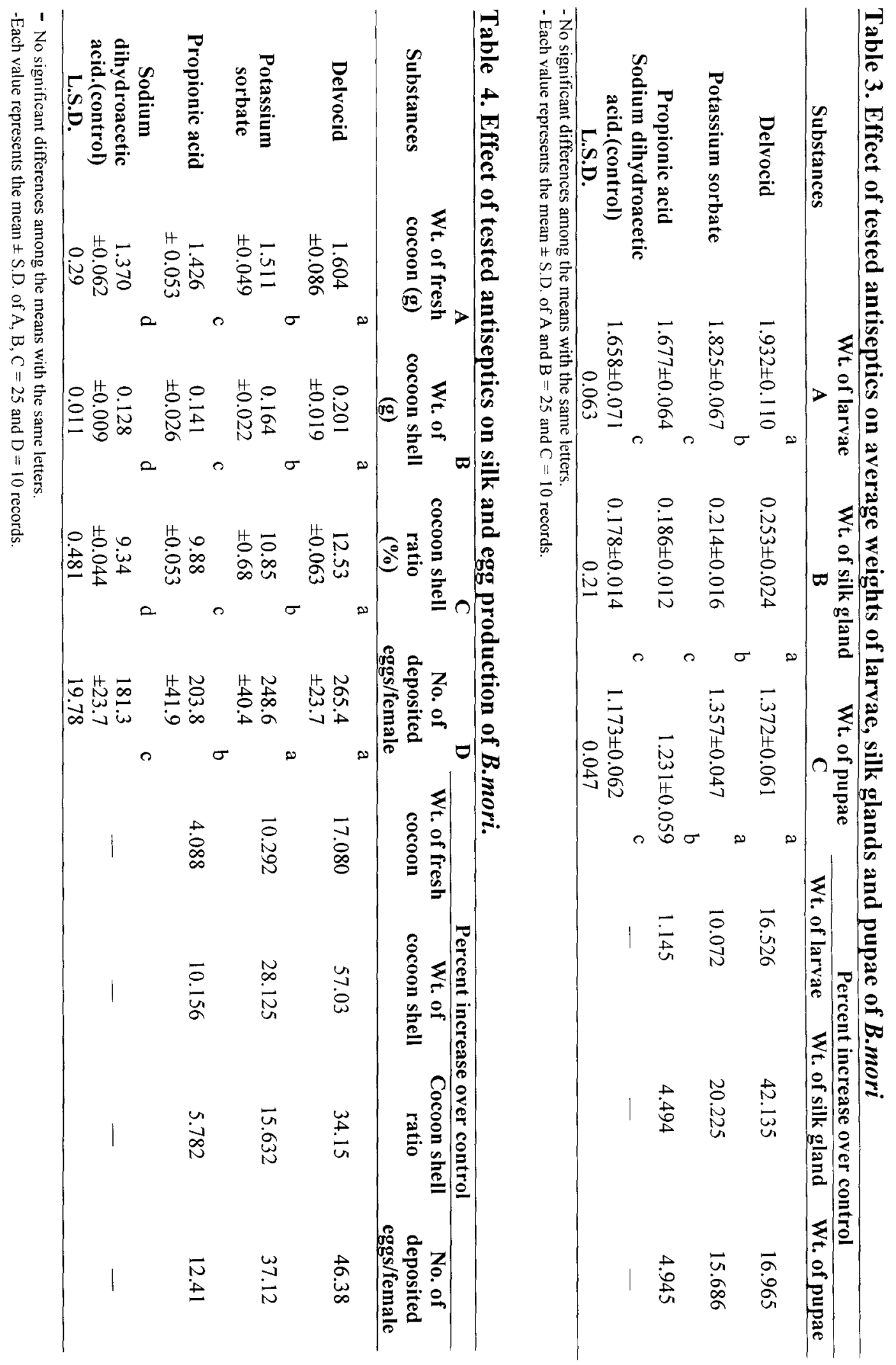


It is worth noting that there was no retardation or acceleration in the duration of $5^{\text {th }}$ larval instar or pupal stage due to the supplemented antiseptics, as well as, no abnormal percentage of the mortality was obtained which were $2.67,2.44,3.10$ and $2.88 \%$ for the treatments of delvocid, potassium sorbate, propionic acid and sodium dihydroacetic acid, respectively.

Effect of tested antiseptics on silk and egg production of silkworm $B$. mori :

As shown in Table 4 the larvae which were fed on supplemented artificial diet with delvocid or potassium sorbate produced heaviest significant fresh cocoons weights (1.604 and 1.511 g.) and cocoon shells (0.201 and 0.164 g.) as well as highest cocoon shell ratio (12.53 and $10.85 \%)$. The lowest parameters of produced cocoons were recognized for both treatments of propionic and sodium dihydroacetic acids. The same trend of results was obtained for the female moth fecundity, since the supplementation of delvocid or potassium sorbate significantly increased the number of deposited eggs per female moth by about 46.38 and $37.12 \%$, in respect more than the treatment of sodium dihydroacetic acid which was the control treatment (Table, 4).

The present results are in agreement with those obtained by Li and Chiang (1982) who reported that the addition of chloramphenicol or sorbic acid to the artificial diet of $B$. mori led to increase the silk production. Liaw et al (1991a) also screened antiseptics for use in artificial diet of $B$. mori by incubation of harmful microorganisms into the diet. Satisfactory antibiosis was found when diet include $10 \mathrm{mg}$. chloramphenical, $5 \mathrm{mg}$. dihdrostreptomycin, $150 \mathrm{mg}$.
Proponic acid and $200 \mathrm{mg}$. sorbic acid per 100g. dry weight. This complex appeared safe for the development of silkworms in addition to raising silk production.

\section{REFERENCES}

Bertleff, W., Roeper, M. And Sava, Y. (2007). Neuro biological effect of interventricular propionic acid in rates. Behavioral Brain Research 176 (1) 149 - 169.

Brummer, J.M. and Stephan, H. (1980). Prevention of molding bread. 1 - use of preservatives and anti - mould agents. Getride, Mehl und Brot 34, (6) 159 - 163 (C.F. Gomaa, 1987).

Cai, Y and Li, S. (1980). Studies on the artificial diet rearing. Sericultural Science China, 6(3) : 181 - 186.

Fukuda, T. (1963). A semi - synthetic diet for Eri - silkworm rearing. Agric. Biol. Chem. 27 (9) : 601 - 609.

Gomaa, E.G. (1987). Effect of chemical preservatives and vegetable oils on the characteristics and shelf life of bread. Minia J. Agric. Res. And Dev. 9 (1) 15 - 28.

Ito, T. (1981). Development of artificial diets and their application in sericulture. Sericologia, 21 (4) : 298 - 306.

Ito, T. And Inokuchi, T. (1992). Nutrition effects of asparagine and glutamine on the silkworm, Bombyx mori L. Applied Entomology and Zoology 27 (4) : 575 - 586.

Li, X. Y. And Chiang, Y. L. (1982). Some factors influencing the larval growth of Bombyx mori L. reared on artificial diet. Acta Entomologia Sinica 25 (4) 382 - 389.

Liaw, G. J, Hsieh, F. K. And Chu, Y. I. (1991 a). Screening of antiseptics for artificial diet for silkworm Bombyx mori L. Chinese Journal of Entomology 11 (3): 180 - 186.

Miao, Yung - Gen and Jiang, Li - Jun (2001). Studies on the minor elements and the antiseptics of artificial diet for silkworm Bombyx mori L. Sericologia 41 (3) 439 - 444. 


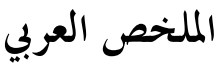

\section{تأثير إضافة بعض المطهرات للغذاء الصناعى على نمو و إنتاجية ديدان حرير القز}

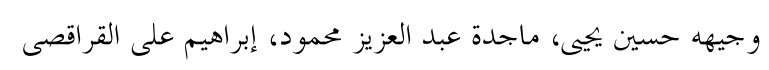

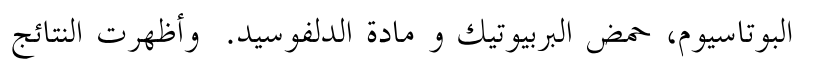

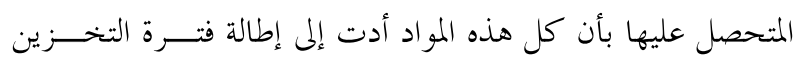

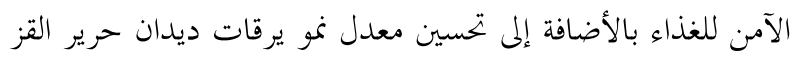

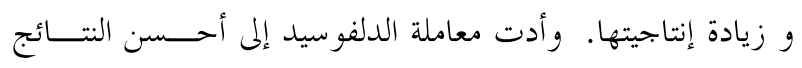

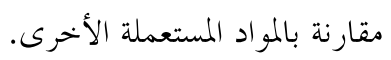

في محاولة للوصول إلى غذاء نصف صناعى محسن هدف زيادة إنتاجية ديدان حرير القز فقد تم إضافة بعض المطهرات إلى الغــــاء النصف صناعى الموصى به وذلك لمنع حدوث الى تلوث بالخمــائر

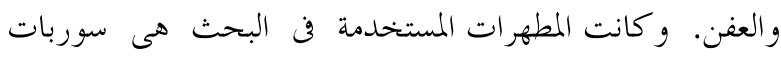

